\title{
Diabetic myonecrosis: an uncommon diabetic complication
}

\section{Punith Kempegowda1,2, Eka Melson 1,2, Gerald Langman², Fady Khattar², Muhammad Karamat ${ }^{2}$ and Quratul-Ain Altaf ${ }^{2}$}

${ }^{1}$ Institute of Metabolism and Systems Research, University of Birmingham, Birmingham, UK and 2University Hospitals Birmingham NHS Foundation Trust, Birmingham, UK
Correspondence should be addressed to P Kempegowda Email

p.kempegowda@nhs.net

\section{Summary}

Diabetic myonecrosis, also known as diabetic muscle infarction is a rare complication of diabetes mellitus usually associated with longstanding suboptimal glycaemic control. Although theories of atherosclerosis, diabetic microangiopathy, vasculitis, ischaemia-reperfusion injury and hypercoagulable state have been proposed to explain the pathophysiology, none of these have been able to individually explain the pathophysiology in entirety. Diabetic renal disease is the most common risk factor for developing DMN and its recurrence. The diagnosis is often missed due to lack of awareness and the presentation mimicking other conditions associated with DM. The routine laboratory investigations are often non-specific and do not provide much value in the diagnosis as well. Muscle biopsy can provide a definite diagnosis but is not currently recommended due to its invasiveness and association with prolonged time to symptoms resolution. Magnetic resonance imaging, in combination with classic history and risk factors can clinch the diagnosis. Treatment is generally analgesia and rest, although the former's use may be limited in the presence of renal disease.

\section{Learning points:}

- Diabetic myonecrosis is a rare complication of diabetes mellitus associated with longstanding suboptimal glycaemic control.

- Diabetic renal disease is a known risk factor, although the evidence is merely observational.

- Although muscle biopsy could provide a definite diagnosis, it is not recommended as it can prolong the disease process and should be reserved only for cases not responding to conventional treatment.

- Typical MRI findings in combination with classic symptoms and risk factors can clinch the diagnosis

- Current treatment recommendations include NSAIDs and/or aspirin (if not contraindicated) alongside bed rest. Physiotherapy is not recommended in the acute phase but should be started as soon as patient is discharged from hospital.

- Optimal glycaemic control is key to prevent recurrence.

\section{Background}

Diabetic myonecrosis (DMN), also known as diabetic muscle infarction, is a rare complication of diabetes mellitus (DM) associated with suboptimal glycaemic control (1). The diagnosis is often missed due to lack of awareness and the presentation mimicking other conditions associated with DM. The routine laboratory investigations are often non-specific and do not provide much value in the diagnosis (2). In this report, we present a young man with longstanding suboptimal diabetes control presenting with thigh pain, swelling and reduced mobility. 


\section{Case presentation}

A 35-year-old South Asian man was admitted with a 3-day history of right thigh pain, swelling and reduced mobility. He did not have any other relevant symptoms and denied any history of trauma. There was also no history of similar symptoms in the past.

He was diagnosed with type 1 diabetes with suboptimal glycaemic control due to long-term poor compliance to treatment. As a result, he had developed bilateral diabetic maculopathy and end-stage kidney disease for which he had recently been initiated on peritoneal dialysis. He was between jobs due to long-term sickness secondary to diabetes at the time of presentation. He is a non-smoker and does not drink any alcohol.

On examination, he was pyrexial and otherwise haemodynamically unremarkable. He had tenderness over the upper third of the anteromedial region of right thigh. There was no erythema or any other signs of inflammation in the area of tenderness. Rest of the clinical examination was unremarkable.

\section{Investigation}

Initial investigations showed anaemia $(92 \mathrm{~g} / \mathrm{L}$; reference range (RR): $133-166 \mathrm{~g} / \mathrm{L})$, neutrophilia $\left(8.3 \times 10^{9} / \mathrm{L}\right.$; RR: $1.6-4.6 \times 10^{9} / \mathrm{L}$ ), increased C-reactive protein (CRP) $(208 \mathrm{mg} / \mathrm{L}$; RR: $0-5 \mathrm{mg} / \mathrm{L})$ and mildly increased creatine kinase (392U/L; RR: 30-200U/L). No signs of infection were seen on chest $\mathrm{X}$-ray and urine analysis. D-dimer was raised ( $1145 \mathrm{ng} / \mathrm{mL}$; RR: $0-243 \mathrm{ng} / \mathrm{mL})$ and the patient underwent ultrasound Doppler to rule out deep vein thrombosis. The scan instead showed extensive diffuse subcutaneous oedema of the right adductor magnus muscle. A differential diagnosis of myositis versus delayed-onset muscle soreness was offered and magnetic resonance imaging (MRI) was recommended. A herniogram performed for suspected leak from the newly inserted peritoneal dialysis catheter showed the tip of the peritoneal dialysis catheter projected over the sacrum and no signs of leak.

\section{Treatment}

The patient was initially treated with intravenous antibiotics and supportive medical therapy for suspected cellulitis. MRI of the right thigh revealed significant muscle oedema with some areas of breaking down suggestive of infective myositis (Fig. 1). The patient continued to experience pain with minimal change in his clinical condition despite 3 weeks of medical treatment. In view of suspected autoimmunity causing myositis, rheumatologists performed extensive autoimmune screen, all of which showed normal results. Orthopaedic opinion was hence sought in view of suboptimal response to medical therapy for 4 weeks. A biopsy of the right thigh muscle was performed and it showed features of
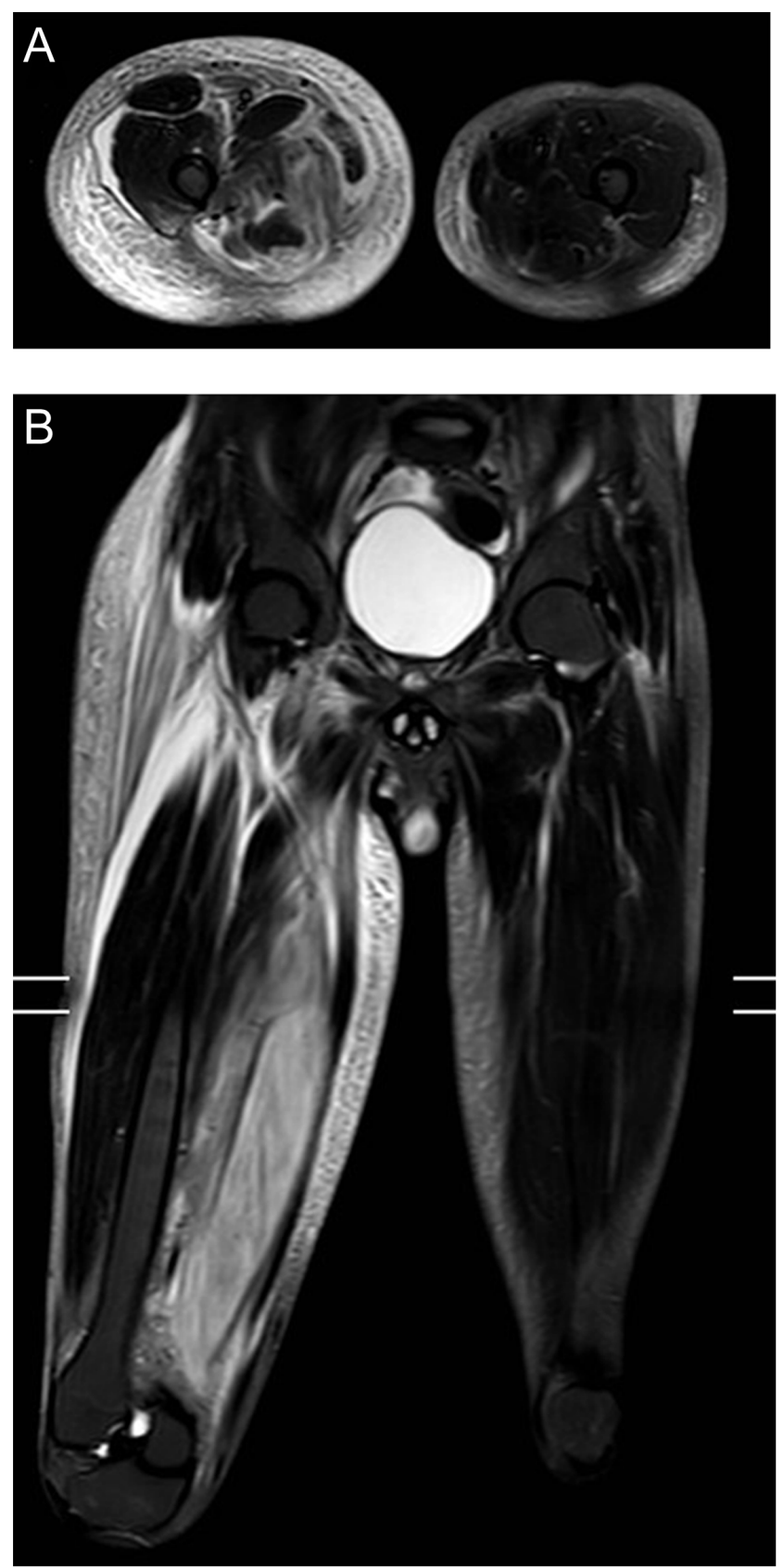

Figure 1

MRI of right lower thigh axial (A) and coronal (B) views: Diffuse oedema of the right adductor muscles, predominantly affecting the adductor magnus muscle with no intramuscular tear or haematoma demonstrated. Picture in keeping with myositis vs delayed-onset muscle soreness (DOMS). 

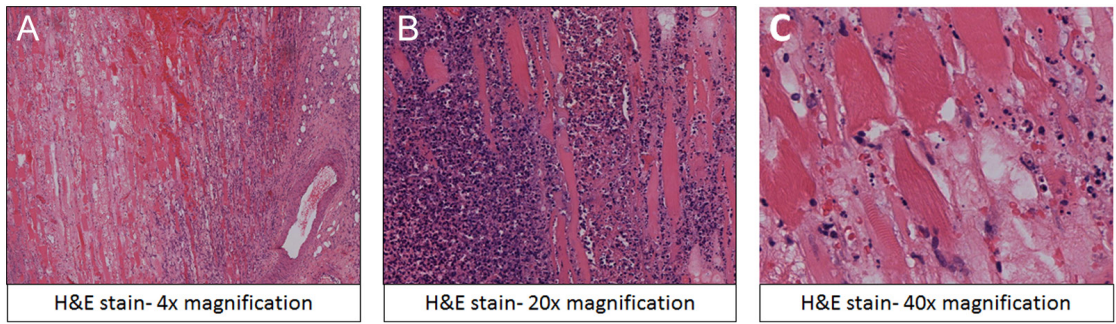

\section{Figure 2}

Histopathology (haematoxylin and eosin stain) of muscle biopsy. (A) $4 \times$ magnification, (B) $20 \times$ magnification, (C) $40 \times$ magnification showing necrotic striated muscle at the left of the image and an inflammatory infiltrate to the right. necrotising myositis (Fig. 2). Five weeks into admission, the patient was referred to the diabetes team to review his suboptimal glycaemic control delaying his recovery. Based on classic history and radiological findings, the patient was diagnosed with DMN. The patient was managed with regular analgesia, bed rest and good glycaemic control.

\section{Outcome and follow-up}

The patient was discharged with advice to continue bed rest and analgesia. He was seen in our outpatient clinic 4 weeks later by which time he was pain-free and awaiting physiotherapy to regain physical strength in the affected leg.

\section{Discussion}

DMN is a rare complication of diabetes mellitus (DM) associated with suboptimal glycaemic control (1). It is mostly seen in patients with type $1 \mathrm{DM}$ but can also occur in type $2 \mathrm{DM}$, with female preponderance in reported literature (3). Although theories of atherosclerosis, diabetic microangiopathy, vasculitis, ischaemia-reperfusion injury and hypercoagulable state secondary to low antithrombin-III levels have been proposed to explain the pathophysiology, none have been entirely convincing (4).

Our case highlights the importance of two major factors that may have influenced the outcome of our case: lack of awareness of DMN amongst medical professionals, highlighted by delayed diagnosis and impact of partial information on imaging requests. In both instances of ultrasound Doppler and MRI, the radiologist was not provided with the patient's history of DM, which may have ruled out DMN as a differential to the image findings. The case also reiterates the importance of early multidisciplinary approach for early diagnosis and treatment.

The common differential diagnoses for DMN are trauma, deep vein thrombosis, hematoma, abscess, fasciitis, inflammatory myopathies, myositis associated with connective tissue diseases, infective myositis including pyomyositis and viral myositis, infiltrating neoplasm, rhabdomyolysis, vascular causes (Behcet disease, sickle cell crisis) and delayed-onset muscle soreness
(DOMS) (5). All of these can be ruled out with detailed history and focused blood and radiological investigations. One other rare differential diagnosis is autoantibody negative immune-mediated necrotizing myopathy. This poorly understood condition tends to affect the younger individuals causing severe weakness in the proximal muscles. However, these lesions have myofibre necrosis with minimal inflammatory cell infiltrate on muscle biopsy, hence differentiating from DMN (6).

Lower limb is the most frequently affected muscle group (87.8\%), with proximal lower limb (above knee) accounting for $58.5 \%$ of all the DMN cases (7). The most common clinical presentation is sudden-onset pain, localised swelling and fever. The diagnosis is often missed at first presentation, due to lack of awareness and these non-specific symptoms. The routine investigations such as white cell count, CK, CRP, ESR and X-ray are often nonspecific to DMN (2).

MRI is currently the investigation of choice, although results are not pathognomonic. Common features in MRI include a hyperintense signal on T2-weighted images and an isointense to hypointense signal on T1-weighted images with associated peri-fascial, peri-muscular and/ or subcutaneous oedema. Ultrasound has also been recommended with findings including a well-marginated, hypoechoic intramuscular lesion. These ultrasound findings can be distinguished from a tumour or necrotic abscess with an absence of internal motion or swirling of fluid with transducer pressure and a lack of predominantly anechoic area in DMN (2).

Muscle biopsy provides definite diagnosis but is not currently recommended due to its invasive nature. Also, biopsy is associated with prolonged time to symptoms resolution in DMN (8). Biopsy, when done early in the presentation, shows areas of necrosis and oedema and late findings include presence of fibrotic tissues and muscle fibre regeneration with lymphocytic infiltration. Currently, biopsy is only reserved for cases that are not responding to conventional treatments.

Non-steroidal anti-inflammatory drugs (NSAIDs) and/or aspirin with bed rest and analgesia are the current recommended treatments for DMN. In one 
study, combination of NSAIDs and aspirin compared to analgesia and bed rest (supportive treatments) improved the recovery time from 57 days to 39 days (9). However, it is important to note that patients with DMN might also have a concurrent renal disease that may limit the use of NSAIDs. We were able to treat our patient with only supportive management in view of his end stage kidney disease; this may have resulted in longer than usual recovery time. Physiotherapy in acute phase of DMN has been controversial, with one study associating physiotherapy with prolonged recovery time from DMN. However, no other studies found such association. The current recommendation is to offer physiotherapy to improve mobility after symptoms resolution $(9,10)$. The evidence for prophylactic anticoagulants in these patients is limited. Other treatment strategies such as glucocorticoid, antibiotics and erythropoietin have been tried in the past with limited efficacy.

Patients with DMN, especially with renal disease, are at high risk of disease recurrence (7). Optimal glycaemic control is considered key to prevent recurrence of DMN. Physiotherapy post-recovery has also been shown to reduce the risk (9). Interestingly, no recurrence was reported in patients who underwent renal transplant, further strengthening the association between diabetes nephropathy and DMN (7). Further studies are needed to study this association.

\section{Patient's perspective}

The patient was frustrated and was on the verge of disengagement from medical care when PK first met him 5 weeks into the patient's hospitalisation. It was understandable the patient was frustrated with ongoing pain and uncertainty of the diagnosis. Following a detailed discussion and explanation of the diagnosis and its pathophysiology, the patient worked along with the diabetes team and improved his glycaemic control, which may have helped with his recovery. He was upbeat in mood and thankful for our help on follow-up in the diabetes outpatient clinic. Following this episode, he has kindly agreed for better engagement with the healthcare system to prevent recurrence.

\section{Declaration of interest}

The authors declare that there is no conflict of interest that could be perceived as prejudicing the impartiality of the research reported.

\section{Funding}

This research did not receive any specific grant from any funding agency in the public, commercial or not-for-profit sector.

\section{Patient consent}

Written informed consent was obtained from the patient.

\section{Author contribution statement}

PK was the initial clinician who diagnosed and managed the case. EM assisted PK in case management, literature review and prepared the first draft of the case. GL performed the histopathology of the biopsy and FK was involved in radiological investigations. MK and QA were the supervising diabetes consultants who oversaw the case management.

\section{References}

1 Angervall L \& Stener B. Tumoriform focal muscular degeneration in two diabetic patients. Diabetologia 19651 39-42. (https://doi. org/10.1007/BF01338714)

2 Trujillo-Santos AJ. Diabetic muscle infarction: an underdiagnosed complication of long-standing diabetes. Diabetes Care 200326 211-215. (https://doi.org/10.2337/diacare.26.1.211)

3 Choudhury BK, Saikia UK, Sarma D, Saikia M, Choudhury SD \& Bhuyan D. Diabetic myonecrosis: an underreported complication of diabetes mellitus. Indian Journal of Endocrinology \& Metabolism 2011 15 S58-S61. (https://doi.org/10.4103/2230-8210.83052)

4 Habib GS, Nashashibi M, Saliba W \& Haj S. Diabetic muscular infarction: emphasis on pathogenesis. Clinical Rheumatology 200322 450-451. (https://doi.org/10.1007/s10067-003-0789-z)

5 Bhasin R \& Ghobrial I. Diabetic myonecrosis: a diagnostic challenge in patients with long-standing diabetes. Journal of Community Hospital Internal Medicine Perspectives 2013 3. (https://doi. org/10.3402/jchimp.v3i1.20494)

6 Pinal-Fernandez I, Casal-Dominguez M \& Mammen AL. Immunemediated necrotizing myopathy. Current Rheumatology Reports 2018 20 21. (https://doi.org/10.1007/s11926-018-0732-6)

7 Yong TY \& Khow KSF. Diabetic muscle infarction in end-stage renal disease: a scoping review on epidemiology, diagnosis and treatment World Journal of Nephrology 20187 58-64. (https://doi.org/10.5527/ win.v7.i2.58)

8 Gupta S, Goyal P, Sharma P, Soin P \& Kochar PS. Recurrent diabetic myonecrosis -an under-diagnosed cause of acute painful swollen limb in long standing diabetics. Annals of Medicine \& Surgery 201835 141-145. (https://doi.org/10.1016/j.amsu.2018.09.003)

9 Horton WB, Taylor JS, Ragland TJ \& Subauste AR. Diabetic muscle infarction: a systematic review. BMJ Open Diabetes Research \& Care 20153 e000082. (https://doi.org/10.1136/ bmjdrc-2015-000082)

10 Morcuende JA, Dobbs MB, Crawford H \& Buckwalter JA. Diabetic muscle infarction. Iowa Orthopaedic Journal 200020 65-74. 